\title{
Impact of COPD in North America and Europe in 2000: subjects' perspective of Confronting COPD International Survey
}

\author{
S. Rennard*, M. Decramer", P.M.A. Calverley ${ }^{\Uparrow}$, N.B. Pride ${ }^{+}$, J.B. Soriano ${ }^{\S}$, P.A. Vermeire ${ }^{f}$, \\ J. Vestbo**
}

Impact of COPD in North America and Europe in 2000: subjects' perspective of Confronting COPD International Survey. S. Rennard, M. Decramer, P.M.A. Calverley, N.B. Pride, J.B. Soriano, P.A. Vermeire, J. Vestbo. C) ERS Journals Ltd 2002.

ABSTRACT: To date, no international surveys estimating the burden of chronic obstructive pulmonary disease (COPD) in the general population have been published. The Confronting COPD International Survey aimed to quantify morbidity and burden in COPD subjects in 2000.

From a total of 201,921 households screened by random-digit dialling in the USA, Canada, France, Italy, Germany, the Netherlands, Spain and the UK, 3,265 subjects with a diagnosis of COPD, chronic bronchitis or emphysema, or with symptoms of chronic bronchitis, were identified.

The mean age of the subjects was 63.3 yrs and $44.2 \%$ were female. Subjects with COPD in North America and Europe appear to underestimate their morbidity, as shown by the high proportion of subjects with limitations to their basic daily life activities, frequent work loss $(45.3 \%$ of COPD subjects of $<65$ yrs reported work loss in the past year) and frequent use of health services $(13.8 \%$ of subjects required emergency care in the last year), and may be undertreated. There was a significant disparity between subjects' perception of disease severity and the degree of severity indicated by an objective breathlessness scale. Of those with the most severe breathlessness (too breathless to leave the house), $35.8 \%$ described their condition as mild or moderate, as did $60.3 \%$ of those with the next most severe degree of breathlessness (breathless after walking a few minutes on level ground).

This international survey confirmed the great burden to society and high individual morbidity associated with chronic obstructive pulmonary disease in subjects in North America and Europe.

Eur Respir J 2002; 20: 799-805.

\begin{abstract}
*University of Nebraska, Pulmonary and Critical Care Medicine Section, Omaha, NE, USA. " Respiratory Division, Catholic University of Leuven, Leuven, Belgium. "University of Liverpool, Dept of Medicine, Clinical Sciences Centre, University Hospital Aintree, Liverpool, UK. ${ }^{+}$Thoracic Medicine, National Heart and Lung Institute, Imperial College School of Medicine, London, UK. ${ }^{\S}$ Worldwide Epidemiology, GlaxoSmithKline Research and Development, Greenford, UK. 'Dept of Respiratory Medicine, University of Antwerp, Antwerp, Belgium. **Dept of Respiratory Medicine, Hvidovre University Hospital, Copenhagen, Denmark.
\end{abstract}

Correspondence: S. Rennard, Pulmonary and Critical Care Medicine Section, University of Nebraska Medical Center, 985125 Nebraska Medical Center, Omaha, NE 68198-5125, USA. Fax: 14025594878

E-mail: srennard@unmc.edu

Keywords: Chronic obstructive pulmonary disease, epidemiology, guidelines, management, subject perspective

Received: December 102001

Accepted after revision: May 12002

This study was supported by GlaxoSmithKline Research and Development, Greenford, UK
Chronic obstructive pulmonary disease (COPD) is a public health problem worldwide [1], and the prevalence of this disease is still increasing. The World Health Organization (WHO) estimates that COPD is currently the twelfth most common cause of morbidity and the sixth leading cause of death in the world. By 2020 , it is estimated to become the fifth most common cause of disability and the third most frequent cause of death, just behind coronary and cerebrovascular disease [2]. Both the direct and indirect economic costs of COPD to society are high $[3,4]$. Tobacco smoking is undoubtedly the most important risk factor for the development of COPD, but not the only one as COPD may also be present

For editorial comments see page 793. among lifelong nonsmokers [5]. Unlike asthma, where public campaigns have increased public awareness and knowledge, COPD is a poorly recognised and often misunderstood condition. Also unlike asthma, on which international surveys were conducted during the 1990s, the International Study on Asthma and Allergies in Childhood in children [6] and the European Community Respiratory Health Survey in adults [7], to date no survey has attempted to obtain worldwide estimates of COPD prevalence or disease impact [8-16].

The importance of COPD is being increasingly recognised by the public health community. A number of national and international guidelines have recommended the ideal management of COPD subjects [17]. Recent papers, however, have highlighted disparities 
among these COPD guidelines [18, 19]. The recently released Global Obstructive Lung Disease guidelines [20], jointly sponsored by the US National Heart, Lung and Blood Institute and the WHO, were developed to provide a more uniform set of recommendations for the diagnosis and management of COPD and to increase subject and public awareness and understanding of this condition [21, 22].

The Confronting COPD International Survey is the first wide-scale international survey using the same methodology to assess the impact of severity, attitude and management of COPD, from both subject and physician perspectives. This report describes the current state of subjects' disability, perception of health, healthcare resource utilisation and quality of respiratory care received related to COPD in North America and Western Europe in 2000.

\section{Methods}

\section{Subject selection}

COPD subjects were identified in the USA, Canada and six European countries (France, Italy, Germany, the Netherlands, Spain and the UK). The study was carried out between August 2000 and January 2001. The survey was conducted by telephone to permit systematic screening of a national sample of households in order to identify a national probability sample of persons who had been diagnosed with COPD. Recent telephone coverage reports indicate that the percentage of households with a telephone in 1999 was $>94 \%$ in the countries included in the survey, with the exception of Germany $(89.3 \%)$ [23]. The sampling method used telephone numbers (listed and unlisted) randomly generated by a truncated listassisted random-digit dialling sampling procedure [24]. Participation was voluntary, confidential and anonymous.

\section{Case definition}

The case definition for COPD was subjects aged $\geqslant 45$ yrs who had a cumulative cigarette consumption of $\geqslant 10$ pack-yrs and who had been diagnosed with COPD, emphysema or chronic bronchitis, or whose symptoms fulfilled a definition of chronic bronchitis, i.e. "persistent coughing with phlegm or sputum from the chest for the last 2 yrs or more". When more than one household member was identified as an eligible COPD subject, a random selection was made to obtain the designated respondent [25].

\section{Data collection}

Telephone interviews were conducted using a structured questionnaire and had a median duration of $25 \mathrm{~min}$. The survey questionnaire [26] was based on the American Thoracic Society questionnaire, which has been previously validated as a tool for the measurement of asthma symptoms in the general population [27], and is highly reliable when administered by telephone [28], with additional questions on healthcare use and activity limitation. Subjects were questioned regarding symptom severity, sleep disruption, overnight hospitalisation, emergency department visits, unscheduled urgent care visits (defined as unexpected visits to the doctor's office, clinic or hospital), activity limitations due to COPD and use of respiratory therapy. The Medical Research Council (MRC) dyspnoea scale, modified according to BESTALL et al. [29], was incorporated into the questionnaire, as a measure of dyspnoea severity, and ranged 5-0 (5: "Too breathless to leave the house"; 4: "Have to stop for breath every few minutes when walking even on level ground"; 3: "Have to stop even when walking at my own pace or walk slower than most people my age"; 2: "Get breathless when hurrying on level ground or walking on slight incline"; 1: "Only get breathless after strenuous exercise"; and 0: "None of these"). Information regarding limitations to their activities of daily living due to COPD were obtained by asking 'How much do you feel your respiratory condition limits what you can do in each of the following areas? - Do you feel it restricts you a lot, some, only a little or not at all in: "Sports and Recreation"; "Normal Physical Exertion"; "Social Activities"; "Sleeping"; "Household Chores"; "Sex Life"; "Family Activities".' Cumulative cigarette consumption (in pack-yrs) was calculated on the basis of the number of cigarettes smoked per day and number of years of daily smoking. The English version of the questionnaire was translated and backtranslated into Dutch, French, German, Italian and Spanish by translators experienced in the use of health surveys. Review by a pulmonologist found no evidence of any significant difference between the original and the backtranslated instrument. Each interview was conducted by experienced interviewers in the mother tongue of the respondent.

\section{Statistical issues}

The study design required $\sim 400$ interviews with COPD subjects in each of the eight countries. This allowed equal sample precision in the population estimates with an error of 5\% and a power of $80 \%$ for a population prevalence of $5 \%$. COPD prevalence was indirectly calculated using household and population weights for each country, based on the third US National Health and Nutrition Examination Survey (NHANES III) [30]. The sample was stratified by region within each country and sampled proportionately; pooled estimates were weighted according to population size. Percentage and quantitative variables were compared using the Chi-squared test and analysis of variance, respectively, and, for variables with a skewed distribution, the Wilcoxon rank sum test. All statistical tests were two-sided and comparisons with a $<5 \%$ probability of error were considered significant. As the major purpose of this survey was to describe the impact of COPD, no corrections for the multiple comparisons made were performed. 


\section{Results}

A total of 201,921 households in the USA, Canada and six countries in Europe were screened and one or more persons aged $\geqslant 45$ yrs with the condition or symptom eligibility for COPD were identified in the household screening in 8,803 households (fig. 1).

Among the 8,314 households in which an individual was confirmed with condition or symptom eligibility, $2,748(33.0 \%)$ failed to meet the smoking criteria for inclusion in the survey. Only $310(5.6 \%)$ qualified respondents refused to conduct the interview, and another $279(5.0 \%)$ persons were deemed too ill to be surveyed. Full interviews were completed with 3,304

General population
87968, no one $\geq 45$ yrs in household
331, other reasons
other reasons
or $\mathrm{CB}_{\text {symptoms }}$

Fig. 1.-Sampling strategy. The stepwise approach to subject screening is shown. The number of contacts "active" at each stage are indicated. The reasons for contacts being dropped from the study and numbers of contacts dropped at each stage are indicated. RDD: random-digit dialling; COPD: chronic obstructive pulmonary disease; $\mathrm{CB}$ : chronic bronchitis.
Table 1.-Demographic and clinical characteristics of included chronic obstructive pulmonary disease (COPD) subjects

\begin{tabular}{lc}
\hline Subjects n & 3265 \\
Females \% & 44.2 \\
Age yrs & $63.3 \pm 10.8$ \\
Retired \% & 54.8 \\
Current smokers \% & 46.2 \\
Cumulative cigarette & $45.2 \pm 35.2$ \\
$\quad$ consumption pack-yrs & \\
Family history of COPD \% & 37.8 \\
Other conditions \% & 48.1 \\
Arthritis & 7.9 \\
Asthma & 3.8 \\
Cancer & 3.2 \\
Diabetes & 6.1 \\
Kidney problems & 11.3 \\
Heart disease & 10.1 \\
Hypertension & 7.5 \\
Self-assessed severity of COPD \% & \\
Mild & 31.8 \\
Moderate & 44.1 \\
Severe & 21.0 \\
MRC dyspnoea score & $2.6 \pm 1.4$ \\
\hline
\end{tabular}

Data are presented as percentage or mean \pm SD. MRC: Medical Research Council. ${ }^{\#}$ : modified according to [29].

of the 5,566 (59.4\%) selected COPD subjects. The demographic and clinical characteristics of the 3,265 COPD subjects included in the survey are presented in table 1 . The mean age of the subjects was $63.3 \mathrm{yrs}$ and $44.2 \%$ were female. Only $46.2 \%$ reported current smoking, with a mean cumulative cigarette consumption of 45.2 pack-yrs and an MRC dyspnoea score of 2.6. Overall, subjects reported as their primary diagnosis COPD (23.1\%), emphysema $(26.2 \%)$ or chronic bronchitis $(36.2 \%)$, with a further $14.5 \%$ identified as undiagnosed subjects who fulfilled the symptomatic definition of chronic bronchitis (fig. 2). There were moderate variations by country in the primary diagnoses. For chronic bronchitis, the scatter was $22-56 \%$ and for emphysema $13-37 \%$ (both $\mathrm{p}<0.05)$.

The survey revealed a prevalence of COPD which was similar in the eight participating countries (fig. 3 ).

Figure 4 presents the perceived severity of the respiratory condition according to the degree of breathlessness as assessed by the MRC dyspnoea scale. It is apparent that many COPD subjects underestimate the severity of their disease and, in individuals objectively scoring 3, 4 and 5 on the MRC dyspnoea scale (at least "Have to stop even when walking at my own pace or walk slower than most people my age"), $75.2 \%, 60.3 \%$ and $35.8 \%$, respectively, considered their disease only mild or moderate. In both female and male participants, the worst dyspnoea was observed in subjects with a diagnosis of COPD or emphysema, followed by chronic bronchitis, whereas undiagnosed subjects who fulfilled the symptomatic definition of chronic bronchitis were the least breathless $(\mathrm{p}<0.05)($ table 2$)$. Females rated their dyspnoea higher than males within each primary diagnosis, despite reporting smoking substantially less than their male counterparts $(\mathrm{p}<0.05)$. 


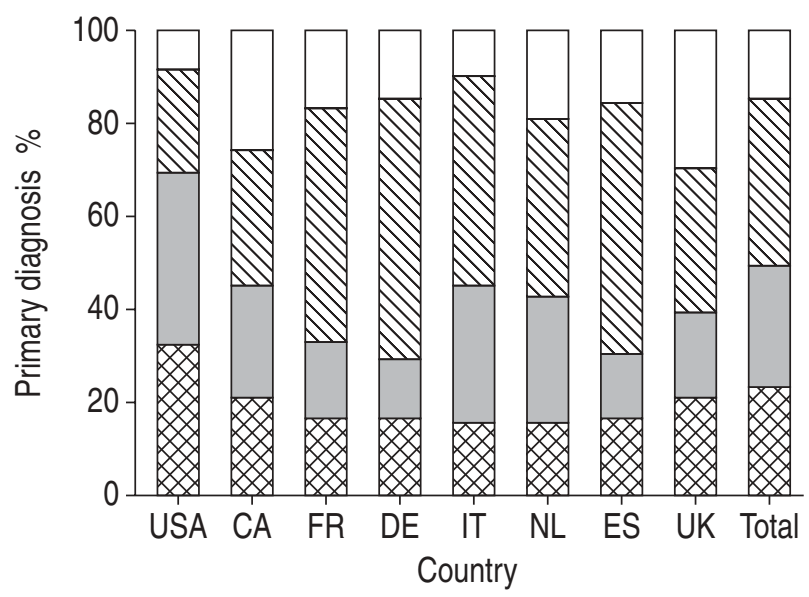

Fig. 2. - Primary diagnosis by country (USA: $n=447$; Canada (CA): $n=401$; France (FR): $n=400$; Germany (DE): $n=400$; Italy (IT): $n=400$; the Netherlands (NL): $n=415$; Spain (ES): $n=402$; UK: $\mathrm{n}=400$; total: $\mathrm{n}=3265$ ). Subjects were eligible for participation if they reported a primary diagnosis of chronic bronchitis $(\mathbb{Q})$, emphysema ( $\square$ ) or chronic obstructive pulmonary disease (1). In addition, if subjects claimed to have chronic cough and sputum production but did not have one of the diagnoses $(\square)$, they were also eligible for participation.

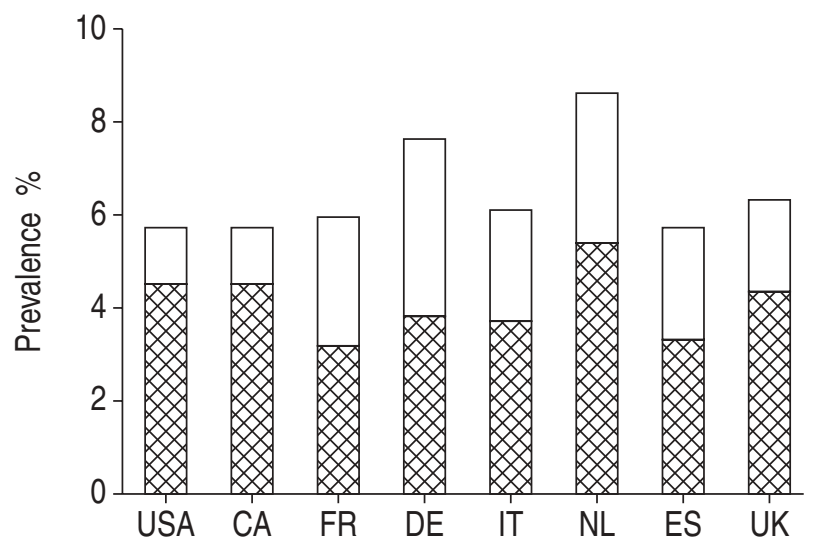

Fig. 3.-Prevalence of chronic obstructive pulmonary disease in subjects ( $\square$ : nonsmokers; $\mathbf{D}$ : smokers) who met the diagnostic criteria by country (USA: $n=11,160$; Canada (CA): $n=12,635$; France (FR): $n=18,165$; Germany (DE): $n=14,904$; Italy (IT): $n=16,270$; the Netherlands (NL): $n=11,081$; Spain (ES): $n=17,387$; UK: $n=12,020)$. The number of households with an individual of $>45$ yrs are indicated. Only individuals with a positive smoking history were included in the statistical analyses (see first paragraph of Results section).

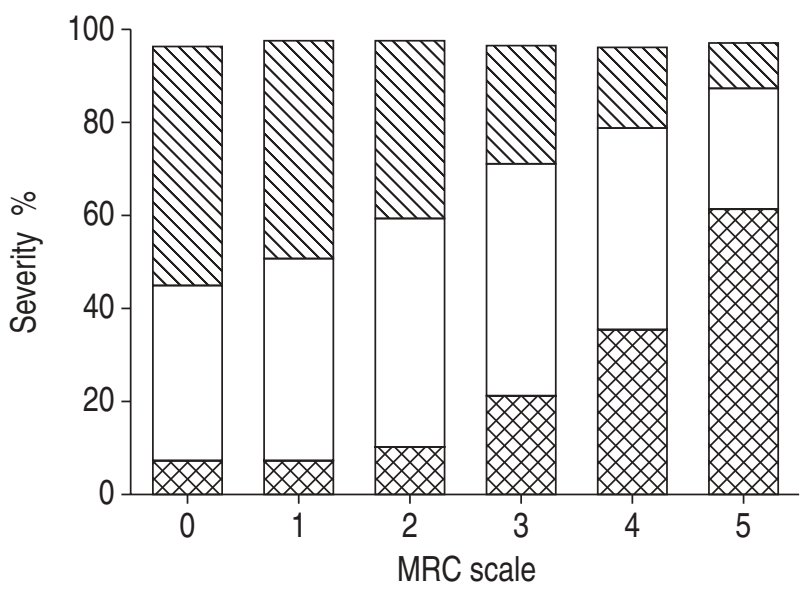

Fig. 4.-Self-reported perceived severity of chronic obstructive pulmonary disease ( $\mathbb{\mathbb { N }}$ : mild; $\square$ : moderate; severe) by degree of breathlessness as assessed by the Medical Research Council (MRC) dyspnoea scale ( 0 (least breathless): $\mathrm{n}=216 ; 1: \mathrm{n}=727 ; 2$ : $\mathrm{n}=898 ; 3: \mathrm{n}=517 ; 4: \mathrm{n}=639 ; 5$ (most breathless): $\mathrm{n}=210$ ) (see Data collection section).

More than a third of persons with COPD (35.7\%) reported that their condition kept them from working, limited their ability to work or had caused them working time loss in the past year. This is likely to be an underestimate since most of these persons were already retired; in subjects of $<65$ yrs, $45.3 \%$ reported work loss during the past year. However, respiratory conditions limited daily life activities in COPD subjects of all ages assessed in the survey (fig. 5).

Middle-aged subjects with COPD suffer a disease burden equal to, if not greater than, that of older subjects; the functional limitations imposed by COPD on persons of $<65$ yrs were reported to be equal to or greater than those on persons of $\geqslant 65 \mathrm{yrs}$ in sports and recreation, social activities, household chores, sex life and family activities. Only in normal physical exertion did significantly fewer persons of $<65$ yrs $(55.7 \%)$ than $\geqslant 65$ yrs $(62.3 \%)$ report limitations as a result of their condition $(\mathrm{p}<0.05)$.

During the year prior to the survey, $12.8 \%$ of participants reported at least one hospitalisation and $13.8 \%$ required emergency care due to COPD. Table 3 summarises the frequency of use of healthcare services and indicators of COPD management, including the frequency of doctor visits, instruction on the use of an

Table 2. - Score on Medical Research Council (MRC) dyspnoea scale and cumulative cigarette consumption according to chronic obstructive pulmonary disease (COPD) diagnostic term and sex

\begin{tabular}{|c|c|c|c|c|c|c|}
\hline & \multicolumn{3}{|c|}{ MRC scale } & \multicolumn{3}{|c|}{ Cumulative cigarette consumption pack-yrs } \\
\hline & Females & Males & p-value $\#$ & Females & Males & p-value \\
\hline All conditions & $2(1-4)$ & $2(1-3)$ & 0.0035 & $30(20-47)$ & $40(23-60)$ & $<0.0000$ \\
\hline COPD & $4(2-4)$ & $3(2-4)$ & 0.0612 & $32(20-50)$ & $40(20-62)$ & 0.0061 \\
\hline Emphysema & $3(2-4)$ & $2(1-4)$ & 0.1050 & $34(20-50)$ & $40(26-60)$ & 0.0014 \\
\hline $\mathrm{CB}$ & $2(1-3)$ & $2(1-3)$ & 0.2815 & $30(19-43)$ & $38(21-59)$ & $<0.0000$ \\
\hline Undiagnosed/symptomatic CB & $2(1-3)$ & $2(1-3)$ & 0.0090 & $30(19-45)$ & $40(25-60)$ & $<0.0000$ \\
\hline
\end{tabular}

Data are presented as median (interquartile range). CB: chronic bronchitis. ${ }^{*}$ : Wilcoxon rank sum test for females versus males. 


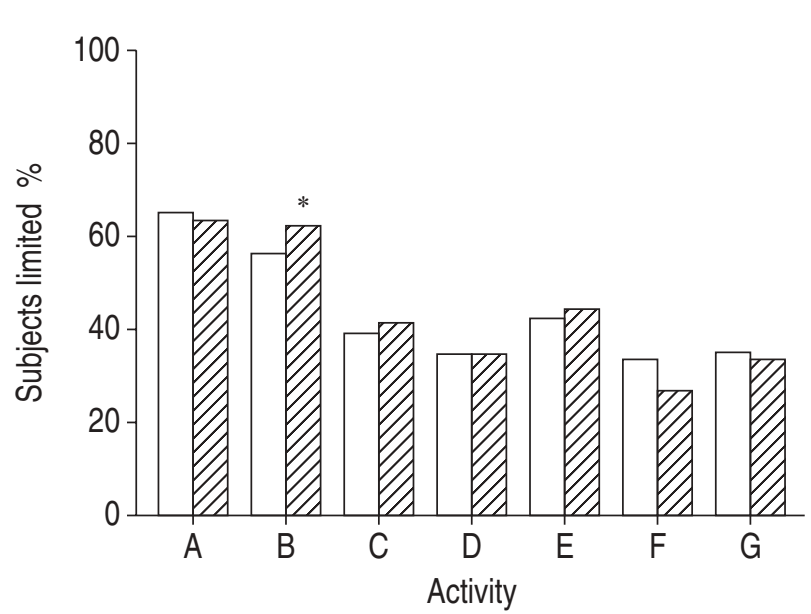

Fig. 5.-Limitation in activities of daily living due to chronic obstructive pulmonary disease in participants of $<65$ yrs $(\square)$ and $\geqslant 65$ yrs $(\mathbb{Z})$. A: sports and recreation; B: normal physical exertion; C: social; D: sleep; E: household chores; F: sex life; G: family. ${ }^{*}: \mathrm{p}<0.05$.

inhaler and the proportion having undergone a lung function test, radiography, computed tomography, pulse oximetry and electrocardiography. Only $67.2 \%$ of the subjects were shown how to use an inhaler, and $45.5 \%$ underwent spirometry. Sixty-one per cent of respondents were taking medication for COPD, and an additional $14 \%$ had taken other inhaled medicines in the past year. These reported medications are detailed in table 3 . The medical speciality of the doctor most involved in the care of the COPD of the participants was general practice in $62.4 \%$ of cases, respiratory medicine in $19.6 \%$ and "others" in $15.0 \%$.

Table 3.-Use of healthcare resources during the past year

\begin{tabular}{lr}
\hline Hospitalisation \% & 12.8 \\
Emergency care \% & 13.8 \\
Frequency of doctor visits about the condition \% & \\
Once a month & 23.8 \\
Every few months & 12.0 \\
At least every 6 months & 29.7 \\
Once a year & 14.7 \\
Never in the past year & 19.2 \\
Shown how to use an inhaler \% & 67.2 \\
Lung function test \% & 78.9 \\
Spirometry & 45.5 \\
Hand-held peak flow & 25.0 \\
Both & 10.5 \\
Chest radiography \% & 56.6 \\
Computed tomography of chest \% & 19.2 \\
Pulse oximetry \% & 41.6 \\
Electrocardiography \% & 47.1 \\
Medication used \% & \\
Short-acting $\beta$-agonist & 25.4 \\
Inhaled corticosteroids & 26.6 \\
Anticholinergics & 17.3 \\
Theophylline & 8.4 \\
Long-acting $\beta$-agonist & 13.4 \\
Leukotriene inhibitors & 2.9 \\
Systemic corticosteroids & 2.8 \\
Nonsteroidal anti-inflammatory & 0.9 \\
\hline
\end{tabular}

\section{Discussion}

The current report describes the burden and impact of COPD in North America and Europe from the perspective of those experiencing the disease. Using telephone-based sampling, smokers with a diagnosis of COPD, emphysema or bronchitis were interviewed, as were smokers with symptoms of chronic bronchitis who had not been formally diagnosed with the disease. Symptoms were common among subjects with both diagnosed and undiagnosed COPD. Although relatively severe dyspnoea was frequently associated with significant compromise of everyday activities that required some exertion, these subjects tended to underestimate this disruption, ranking their disease as mild or moderate. Symptoms tended to be equally reported among younger subjects and those who were older and retired. In addition, females reported prevailing symptoms that affected daily life nearly as often as males, although reporting a history of smoking less often than males in the study. Finally, a substantial number of subjects with both symptoms and a diagnosis reported they were not instructed on the proper use of prescribed inhalers, and even fewer reported ever having undergone spirometry testing, as recommended in current guidelines. Taken together, these results indicate that COPD is very prevalent, has a major impact on affected subjects, is underestimated with regard to its impact on subjects and is probably underdiagnosed and undertreated.

The methods used in the current survey have both strengths and limitations. The study was not designed as a prevalence survey. Nevertheless, based on the information obtained, the prevalence of COPD for the population of smokers aged $\geqslant 45 \mathrm{yrs}$ in the countries surveyed is estimated to be $4 \%$, ranging from $3.2 \%$ in France to $5.4 \%$ in the Netherlands. This estimate of prevalence for COPD across the countries surveyed is much more uniform than that obtained for asthma in the Asthma Insights and Reality in Europe study, which used similar methods and obtained estimates ranging $2.5-15.2 \%$ [31]. It is quite comparable with the prevalence estimates obtained in NHANES III [32], which estimated diagnosed COPD to have a prevalence of $3.1 \%$, asthma of $2.7 \%$ and undiagnosed airflow obstruction of $12.0 \%$. The limitations of random-digit dialling have been discussed elsewhere [24]. One limitation is the availability of telephone lines. In the countries surveyed, $\geqslant 89 \%$ of households have telephones, obviously excluding those without telephone lines from the study. COPD prevalence increases with decreasing socioeconomic status. Thus the prevalence of COPD is likely to have been underestimated to the degree that sampling bias was introduced on the basis of telephone access in the current survey. It is also likely that elderly subjects residing in chronic care facilities were undersampled. Finally, the methods used in the current survey do not permit independent verification of self-reported information. Nevertheless, consistency across the various countries evaluated provides internal validation of the observations made.

The methodology used in the current survey differs from that used in previous epidemiological studies of 
COPD and provides a slightly different view of the COPD subject population. Telephone sampling permits nonclustered sampling of units, eliminates interviewer control over sample selection and provides anonymity for the respondent. The sampling method used, therefore, eliminates biases introduced by other surveys in which subjects may be identified based on referral to a specialist or for pulmonary function testing. Results with such subjects are probably skewed towards increased severity. Indeed, the majority of subjects surveyed received their care primarily from general practitioners and one in five reported never having undergone any lung function testing. Despite this, disease severity, as indicated by breathlessness and disability, was high.

The current survey identified subjects based on their self-reported diagnosis. It is likely that, in some cases, asthma was misclassified as COPD. The findings of the current survey, however, are unlikely to be dominated by subjects with asthma for several reasons. First, the inclusion criteria of age $(\geqslant 45 \mathrm{yrs})$ and smoking $(\geqslant 10$ pack-yrs $)$ were intended to minimise misclassification with asthmatics. It is of note that nearly a third of eligible subjects were excluded because they lacked sufficient smoking history. Although many of these individuals may have had asthma, the survey results are also consistent with a substantial prevalence of COPD in nonsmokers. This finding is consistent with other studies indicating that $15-20 \%$ of COPD subjects are lifelong nonsmokers. In addition, the current study utilised the same methodology as used recently in a survey of asthma [31]. Several findings in the current survey differ importantly from that of the asthma survey, including the high prevalence of dyspnoea and disability. One striking finding further highlighting the severity of functional compromise in COPD subjects is speech difficulty. Subjects required $\geqslant 25$ min to complete the survey. Due to breathlessness associated with talking, it was difficult for some respondents to complete the interview in a single session and 279 were excluded because they were too ill to answer the questionnaire. Speech problems were not observed in the asthma survey.

The current survey also identified subjects with symptoms of chronic cough and sputum production. It is possible to solicit information about such symptoms over the telephone, although not to determine the presence or absence of airflow limitation. Current COPD guidelines include individuals with these symptoms in the spectrum of COPD patients [20]. Such individuals would be at least stage 0 and may be of more advanced stage, depending on the results of lung function testing. Such individuals, moreover, would have been included in the traditional definition of "chronic bronchitis" independent of airflow limitation. Their inclusion in the current survey, therefore, is consistent with the purpose of defining the spectrum and impact of COPD as a whole. Without doubt, subsets of patients with COPD can be identified in whom specific features may be more prominent than in others.

The current survey challenges the stereotypic image of the COPD subject, an elderly male smoker compromised by dyspnoea. Females represented nearly half of the COPD subjects in the current survey. It is possible that the telephone sampling methodology used in the current survey resulted in overrepresentation of females as females have been suggested to be more likely to answer the telephone. The females in the current survey, interestingly, reported less intense smoking histories than the males. This is consistent with the possibility that females are more susceptible to the deleterious effect of tobacco smoke than males [33]. Dyspnoea was common and was more prevalent in older COPD subjects. Daily activities were also compromised in COPD subjects. These problems were highly prevalent among younger COPD subjects.

Current guidelines recommend diagnostic assessment of COPD subjects, including measurement of lung function. Aggressive use of bronchodilators is recommended as this can significantly improve both lung function and symptoms. Use of bronchodilators, however, requires adequate subject instruction on the use of these medications. The current survey was not designed to assess compliance with current guidelines. Nevertheless, the large numbers of subjects who had not undergone diagnostic testing and who had not received training in inhaler use suggest that the key recommendations of current guidelines are far from being uniformly implemented. The results are also consistent with both underdiagnosis and presumably undertreatment of the COPD population. In addition, the survey was not designed to assess compliance with therapy as recommended by the guidelines. Nevertheless, by self-report, subjects were apparently undertreated with bronchodilators compared to current guidelines [20]. Conversely, use of other medications was reported as being somewhat greater than might be anticipated from application of these guidelines. The accuracy, however, of the self-reported medication use obtained in the current survey is uncertain. Nevertheless, the reported data suggest that considerable improvement in the management of COPD patients may be possible.

In summary, chronic obstructive pulmonary disease subjects in the population appear to suffer from relatively severe dyspnoea and disability despite often regarding their disease as mild to moderate. Chronic obstructive pulmonary disease subjects, moreover, may be both underdiagnosed and undertreated in North America and in the six Western European countries surveyed. Although the magnitude of the public health problem presented by chronic obstructive pulmonary disease is becoming increasingly recognised in the public health community, public awareness of chronic obstructive pulmonary disease and implementation of currently recommended diagnostic and therapeutic modalities need to be improved.

\footnotetext{
Acknowledgements. The authors would like to thank J. Boyle for consultation regarding the survey methods and statistics and L. Richards and M. Tourek for assistance with manuscript preparation.
} 


\section{References}

1. World Health Organization. The World Heath Report 1998. Life in the 21st Century. A Vision for All. Geneva, World Health Organization, 1998.

2. Murray CJ, Lopez AD. Alternative projection of mortality by cause 1990-2020: global burden of disease study. Lancet 1997; 349: 1498-1504.

3. Friedman M, Hilleman DE. Economic burden of chronic obstructive pulmonary disease. Impact of new treatment options. Pharmacoeconomics 2001; 19: 245 254.

4. Sullivan SD, Ramsey SD, Lee TA. The economic burden of COPD. Chest 2000; 117: 5S-9S.

5. Silverman EK, Speizer FE. Risk factors for the development of chronic obstructive pulmonary disease. Med Clin North Am 1996; 80: 501-522.

6. International Study of Asthma and Allergy in Childhood. Worldwide variations in the prevalence of asthma symptoms: the International Study of Asthma and Allergy in Childhood (ISAAC). Eur Respir J 1998; 12: 315-335.

7. Burney $\mathrm{P}$, Chinn S, Jarvis D. Variations in the prevalence of respiratory symptoms, self-reported asthma attacks, and use of asthma medications in the European Community Respiratory Health Survey. Eur Respir J 1996; 9: 687-695.

8. Gulsvik A. Mortality in and prevalence of chronic obstructive pulmonary disease in different parts of Europe. Monaldi Arch Chest Dis 1999; 54: 160-162.

9. Kim N, Leeper KV. Epidemiology of chronic bronchitis and acute infective exacerbations of chronic bronchitis. Semin Respir Crit Care 2000; 21: 73-78.

10. Chen JC, Mannino DM. Worldwide epidemiology of chronic obstructive pulmonary disease. Curr Opin Pulm Med 1999; 5: 93-99.

11. Hurd S. The impact of COPD on lung health worldwide: epidemiology and incidence. Chest 2000; 117: $1 \mathrm{~S}-4 \mathrm{~S}$

12. British Lung Foundation. Chronic obstructive pulmonary disease. London, British Lung Foundation, 1999.

13. Lynn J, Ely EW, Zhong Z, et al. Living and dying with chronic obstructive pulmonary disease. $J$ Am Geriatr Soc 2000; 48: S91-S100.

14. Rossi A, Confalonieri M. Burden of chronic obstructive pulmonary disease. Lancet 2000; 356: Suppl., s56.

15. Viegi G, Scognamiglio A, Baldacci S, Pistelli F, Carrozzi L. Epidemiology of chronic obstructive pulmonary disease (COPD). Respiration 2001; 68: 4-19.

16. Anto JM, Vermeire P, Vestbo J, Sunyer J. Epidemiology of chronic obstructive pulmonary disease. Eur Respir J 2001; 17: 982-994.

17. Fabbri L, Caramori G, Beghe B, Papi A, Ciaccia A. Chronic obstructive pulmonary disease international guidelines. Curr Opin Pulm Med 1998; 4: 76-84.

18. Lacasse Y, Ferreira I, Brooks D, Newman T, Goldstein RS. Critical appraisal of clinical practice guidelines targeting chronic obstructive pulmonary disease. Arch Intern Med 2001; 161: 69-74.

19. Grossman RF. Use of guidelines and risk stratification in acute exacerbations of chronic obstructive pulmonary disease. Semin Respir Crit Care 2000; 21: 113-122.

20. Pauwels RA, Buist AS, Calverley PM, Jenkins CR, Hurd SS. Global strategy for the diagnosis, management, and prevention of chronic obstructive pulmonary disease. NHLBI/WHO Global Initiative for Chronic Obstructive Lung Disease (GOLD) Workshop summary. Am J Respir Crit Care Med 2001; 163 : 1256-1276.

21. Hurd S. International efforts directed at attacking the problem of COPD. Chest 2001; 117: 336S-338S.

22. Gross NJ. The GOLD standard for chronic obstructive pulmonary disease. Am J Respir Crit Care Med 2001; 163: 1047-1048.

23. International Telecommunications Union. World Telecommunications Indicators: 2000-2001. Geneva, International Telecommunications Union, 2001.

24. Potthoff RF. Telephone sampling in epidemiologic research: to reap the benefits, avoid the pitfalls. Am $J$ Epidemiol 1994; 139: 967-978.

25. Waksberg J. Sampling methods for random digit dialing. J Am Stat Assoc 1978; 73: 40-46.

26. Ferris BG. Epidemiology standardization project. Am Rev Respir Dis 1979; 118: 1S-120S.

27. Toren J, Brisman J, Jarvholm B. Asthma and asthmalike symptoms in adults assessed by questionnaires. Chest 1993; 104: 600-608.

28. Galobardes B, Sunyer J, Anto JM, Castellsague J, Soriano JB, Tobias A. Effect of the method of administration, mail or telephone, on the validity and reliability of a respiratory health questionnaire. The Spanish Centers of the European Asthma Study. J Clin Epidemiol 1998; 51: 875-881.

29. Bestall JC, Paul EA, Garrod R, Garnham R, Jones PW, Wedzicha JA. Usefulness of the Medical Research Council (MRC) dyspnoea scale as a measure of disability in patients with chronic obstructive pulmonary disease. Thorax 1999; 54: 581-586.

30. Mannino DM, Gagnon RC, Petty TL, Lydick E. Obstructive lung disease and low lung function in adults in the United States: data from the National Health and Nutrition Examination Survey, 19881994. Arch Intern Med 2000; 160: 1683-1689.

31. Rabe KF, Vermeire PA, Soriano JB, Maier WC. Clinical management of asthma in 1999: the Asthma Insights and Reality in Europe (AIRE) study. Eur Respir J 2000; 16: 802-807.

32. Coultas DB, Mapel D, Gagnon R, Lydick E. The health impact of undiagnosed airflow obstruction in a national sample of United States adults. Am J Respir Crit Care Med 2001; 164: 372-377.

33. Prescott E, Bjerg AM, Andersen PK, Lange P, Vestbo J. Gender difference in smoking effects on lung function and risk of hospitalization for COPD: results from a Danish longitudinal population study. Eur Respir J 1997; 10: 822-827. 